\title{
Stretch-Induced Helical Conformations in Poly(L-lysine)/Hyaluronic Acid Multilayers
}

Sarah Zahouani, ${ }^{\dagger, \dagger}$ Alain Chaumont, ${ }^{\S}$ Bernard Senger, ${ }^{\dagger, \downarrow}$ Fouzia Boulmedais, ${ }^{\prime / \perp}$ Pierre Schaaf, ${ }^{\dagger, \ldots, /, \perp, *}$ Loïc Jierry, ${ }^{\prime / \perp}$ and Philippe Lavalle ${ }^{\dagger, t, *}$

† Institut National de la Santé et de la Recherche Médicale, INSERM Unité 1121, 11 rue Humann, 67085 Strasbourg Cedex, France.

\$ Faculté de Chirurgie Dentaire, Université de Strasbourg, 8 rue Sainte Elisabeth, 67000 Strasbourg, France.

$\S$ Faculté de Chimie, UMR 7177, Université de Strasbourg, 1 rue Blaise Pascal, 67008 Strasbourg Cedex, France.

// Institut Charles Sadron, CNRS UPR 22, 23 rue du Loess, 67034 Strasbourg Cedex, France.

$\perp$ University of Strasbourg Institute of Advanced Study, 5 allée du Général Rouvillois, 67083 Strasbourg Cedex, France.

*Corresponding authors: schaaf@ unistra.fr, philippe.lavalle@inserm.fr 


\section{SUPPORTING INFORMATION}
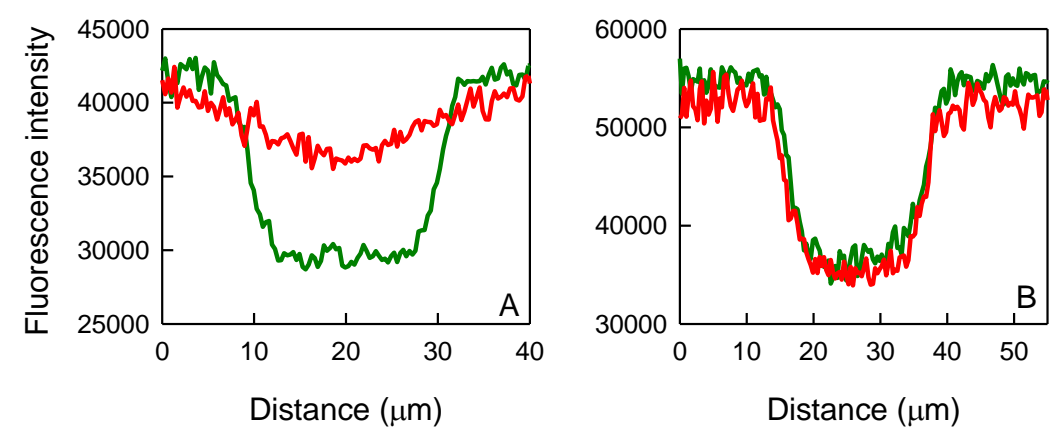

Figure S1. FRAP experiments aimed at comparing the mobility of FITC-labeled PLL chains within a non-cross-linked (A) and a cross-linked (B) (PLL/HA) 23 film. A circular region of interest (10.6 $\mu \mathrm{m}$ in radius $)$ in the image $(84.9 \mu \mathrm{m} \times 84.9 \mu \mathrm{m})$ is bleached at time 0 . The recovery is monitored over a few min. The intensity profiles are shown at $0.7 \mathrm{~s}$ and $150 \mathrm{~s}$ after bleaching and reveal unambiguously the strong reduction of the mobility due to the cross-linking of the film.

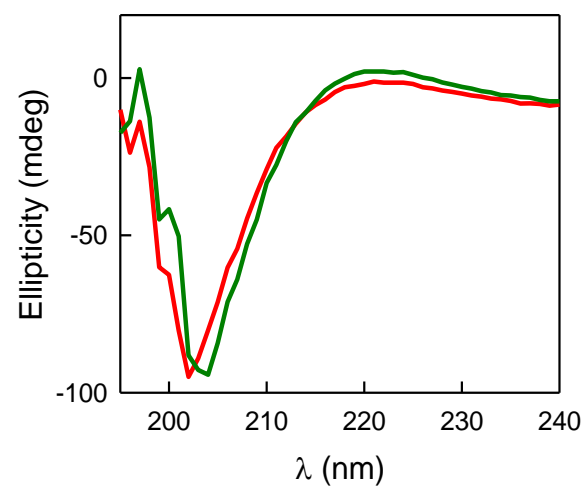

Figure S2. Representative CD spectra of cross-linked (red curve) and non-cross-linked (green curve) $(\mathrm{PLL} / \mathrm{HA})_{23}$ films in the non-stretched state $(\varepsilon=0 \%)$. 


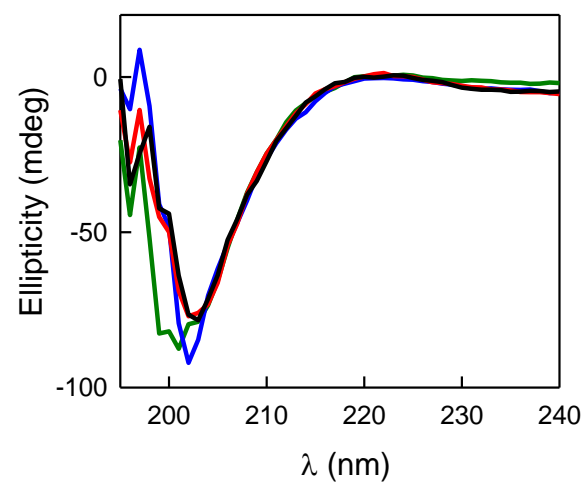

Figure S3. CD spectra of a cross-linked (PLL/HA) 23 film in the non-stretched state $\varepsilon=0 \%$ (green curve) and brought back to the non-stretched state after strains of $\varepsilon=20 \%$ (blue curve), $40 \%$ (red curve), $60 \%$ (black curve).

A

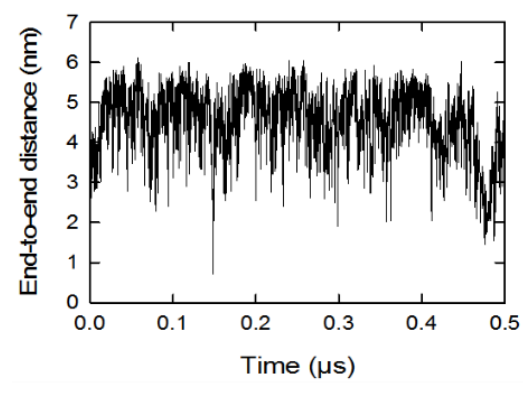

B

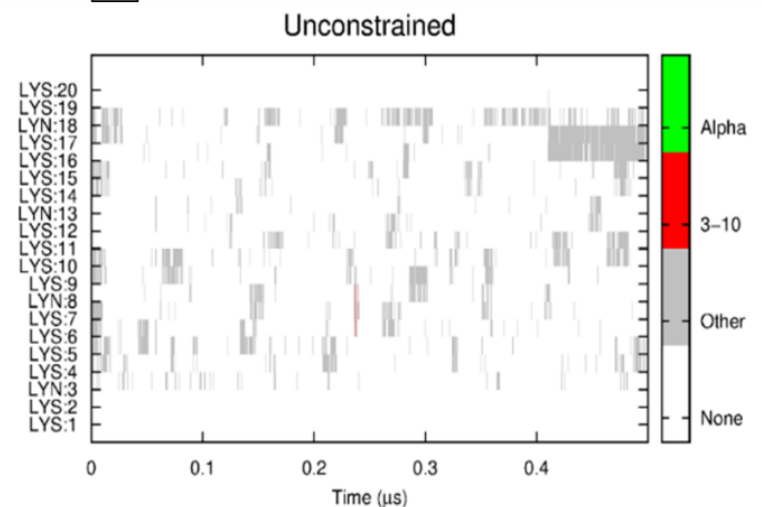

Figure S4. Variation of the distance between the first and the $20^{\text {th }}$ residue of a PLL20 chain as a function of time (A) and associated secondary structures (B) obtained by Molecular Dynamics (MD) of a free PLL20 chain in water. Neither $\alpha$-helices nor 310 -helices appear over $500 \mathrm{~ns}$ simulation time in the non-constrained chain. 

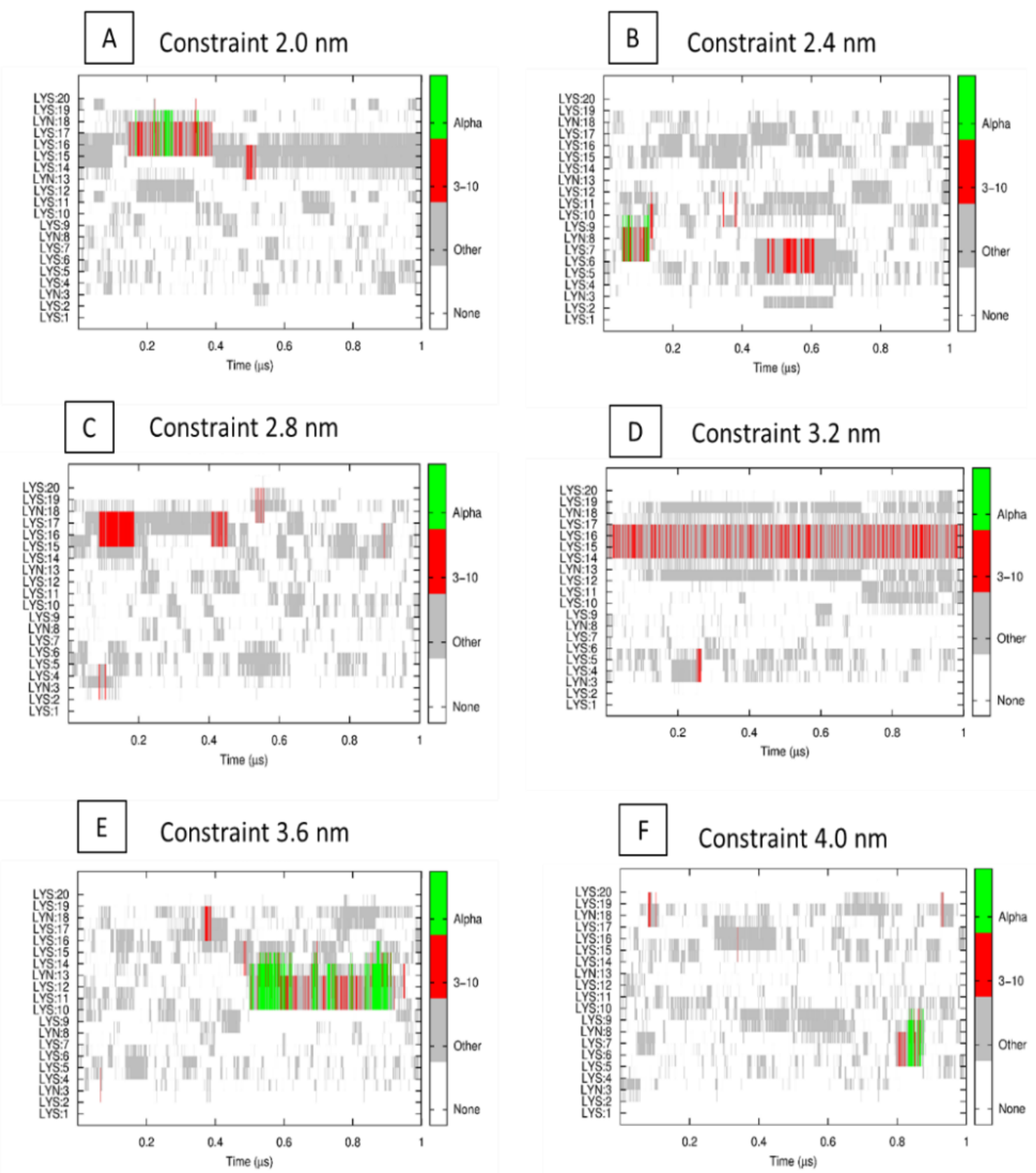

Figure S5. Secondary structures of PLL20 as a function of time predicted by Molecular Dynamics of a PLL20 chain in water. The end-to-end distance is fixed to 2.0 (A), 2.4 (B), 2.8 (C), 3.2 (D), 3.6 (E) and 4.0 (F) $\mathrm{nm}$. $\alpha$-helices and 310 -helices appear over $1 \mu \mathrm{s}$ simulation time in these constrained chains. 
CD spectrum of a cross-linked, non-stretched PLL/HA film at pH 7.4 or 11.6. The CD spectrum of a cross-linked, non-stretched PLL/HA film at $\mathrm{pH} 11.6$ has been shown in Figure 2 (red curve) of the main text. The CD spectra of the PLL in solution either in the $\alpha$-helix conformation or in the $\beta$-sheet conformation or in the random coil conformation were shown in Figure 1 of the main text. The CD spectrum of the HA corresponding to unordered conformations was also given in Figure 1 of the main text. In order to fit the experimental spectrum of the film at $\mathrm{pH} 11.6$, we tried to combine the three spectra corresponding to the three aforementioned PLL structures and the HA spectrum. The resulting spectrum is a weighted sum of the four individual spectra, the weights being obtained by a linear least squares method. As appears in Figure S6a, the agreement is quite poor, especially in the region of the minimum located at about $205 \mathrm{~nm}$. It follows that the CD spectrum of the PLL/HA film is not merely a linear combination of the spectra mirroring the possible structures of its isolated constituents. In contrast, the linear combination of the same PLL random coil spectrum and of the same HA spectrum leads to a quite good reproduction of the nonstretched cross-linked film spectrum at pH 7.4 (Figure S6b).
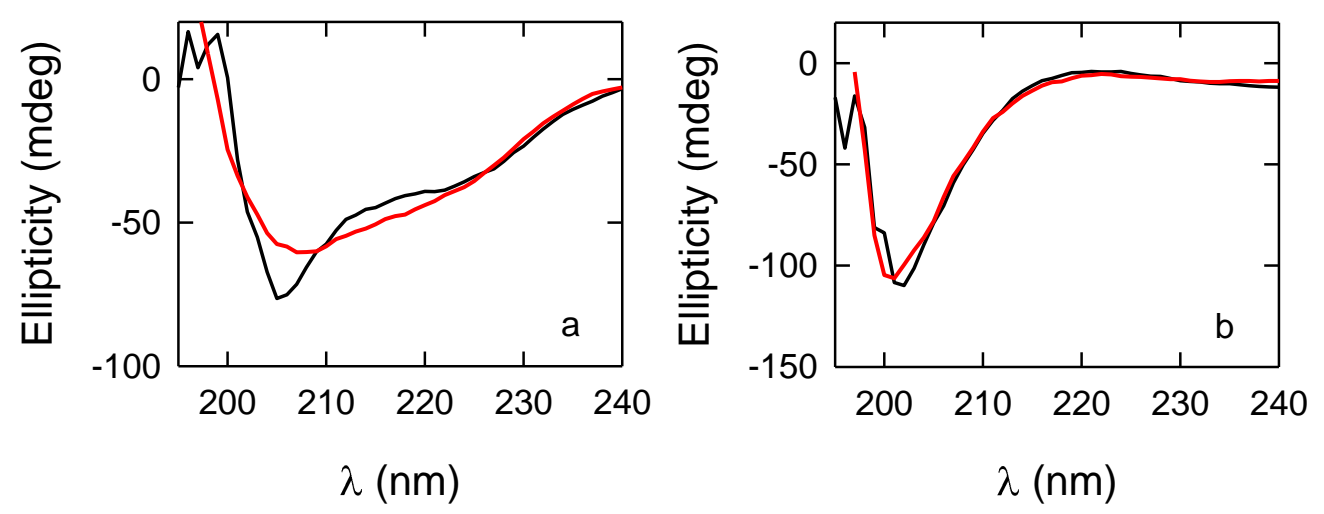

Figure S6. CD spectrum of a cross-linked (PLL/HA) $)_{23}$ film in the non-stretched state $(\varepsilon=0 \%)$ at pH 11.6 (a) and 7.4 (b),both at room temperature (black line). Optimum linear combination (red line) of the spectra corresponding to the PLL $\alpha$-helix conformation, $\beta$-sheet conformation and random coil conformation, as well as to the HA. 
Computation of the weighted average of the wavelength and of the skewness. The weighted average of the wavelength $\langle\lambda\rangle$ of a CD spectrum between the bounds $\lambda_{1}$ and $\lambda_{2}$ is obtained using:

$$
<\lambda>=\frac{\int_{\lambda_{1}}^{\lambda_{2}} \lambda|\theta(\lambda)| \mathrm{d} \lambda}{\int_{\lambda_{1}}^{\lambda_{2}}|\theta(\lambda)| \mathrm{d} \lambda}
$$

where $\theta$ represents the ellipticity. The weighted central moment of order $k(k \geq 1)$ is given by:

$$
m_{k}=\frac{\int_{\lambda_{1}}^{\lambda_{2}}(\lambda-<\lambda>)^{k}|\theta(\lambda)| \mathrm{d} \lambda}{\int_{\lambda_{1}}^{\lambda_{2}}|\theta(\lambda)| \mathrm{d} \lambda}
$$

Then the skewness is defined by the following combination of $m_{2}$ and $m_{3}$ (Abramowitz, M. and Stegun, I. Handbook of Mathematical Functions, p. 928. Dover Publications, Inc., New York, 1970):

$$
\gamma_{1}=\frac{m_{3}}{m_{2}^{3 / 2}}
$$

